\title{
INFLUÊNCIA DE FATORES ANÁTOMO-FISIOLÓGICOS NA MEDIDA INDIRETA DA PRESSÃO ARTERIAL: IDENTIFICAÇÃO DO CONHECIMENTO DOS ENFERMEIROS
}

\author{
Thelma Leite de Araujo* \\ Edna Apparecida Moura Arcuri**
}

\begin{abstract}
ARAUJO,T.L. de; ARCURI, E.A.M. Influência de fatores anátomo-fisiológicos na medida indireta da pressão arterial: identificação do conhecimento dos enfermeiros. Rev.latino-am.enfermagem, Ribeirão Preto, v. 6, n. 4, p. 21-29, outubro 1998.
\end{abstract}

O estudo foi realizado com enfermeiros de hospitais de cardiologia e teve como objetivo caracterizar o conhecimento sobre os aspectos conceituais e fatores anátomo-fisiológicos que influenciam a medida indireta da pressão arterial.

UNITERMOS: medida indireta da pressão arterial, esfigmomanometria

\section{INTRODUÇÃO}

A continuidade da vida ocorre graças à manutenção do aporte de oxigênio necessário aos tecidos e à remoção dos produtos catabólicos, o que é garantido pela preservação das condições hemodinâmicas. A pressão arterial (PA), produto do débito cardíaco e da resistência dos vasos periféricos, é elemento fundamental da dinâmica sangüínea e para garantir a nutrição tecidual é necessário que a tensão na parede arterial atinja determinados níveis. Assim, a PA é um dos mais importantes parâmetros de avaliação do sistema cardiovascular, razão por que a sua mensuração é uma das práticas mais comuns na avaliação clínica.

Considerando as situações de assistência primária, secundária e terciária ocorridas nos domicílios, unidades sanitárias, unidades de cuidados intensivos, recuperações anestésicas e cirúrgicas, pode-se mesmo afirmar que, a cada minuto das 24 horas, centenas ou mesmo milhares de profissionais da área de enfermagem encontram-se executando o procedimento de verificação da pressão arterial.

Como a PA retrata as condições funcionais do sistema circulatório, a precisão na sua medida vem-se apresentando como um grande desafio, caracterizado pelas tentativas de diminuir os erros introduzidos pelo observador (pessoa que registra a PA, geralmente enfermeiro e médico), pelo próprio cliente, que pode apresentar aumento dos níveis da pressão arterial devido ao procedimento da medida e a presença do profissional médico, e pelo instrumental utilizado, geralmente escolhido e mantido pelo enfermeiro.

A literatura tem destacado o enfermeiro como um profissional que causa menor reação de alerta no cliente (MANCIA et al., 1987; PIERIN, 1992). A valorização desse profissional teve como marco inicial o estudo desenvolvido por WILCOX (1961), o primeiro a demonstrar a magnitude do erro do observador no registro da pressão arterial sistólica (PAS) e diastólica (PAD). Torna-se assim imperativa a necessidade de o profissional enfermeiro detectar dados hemodinâmicos precisos e interpretá-los com segurança, o que requer um sólido conhecimento teórico e prático no campo da esfigmomanometria, a fim de garantir a tomada de decisão e encaminhamentos corretos. Apesar dessa assertiva, o que se observa é um desconhecimento preocupante, exceto para alguns aspectos da habilidade motora. A medida da PA vista como um procedimento corriqueiro, não é abordada em programas de educação continuada ou em cursos de atualização, sendo que pouco é acrescentado ao que se aprende durante o curso de graduação.

GAGNÉ (1980) considera que a obtenção do conhecimento ocorre como resultado de uma aprendizagem definida como sendo o processo pelo qual os seres humanos se tornam capazes de modificar seu comportamento, podendo-se dizer que houve aprendizagem quando se observa mudança comportamental e permanência dessa mudança. Para o autor os resultados da aprendizagem são as capacidades humanas que tornam possível uma variedade de desempenho; são elas: informações verbais, habilidades

\footnotetext{
* Enfermeira. Professor Doutor do Departamento de Enfermagem da Universidade Federal do Ceará

** Enfermeira. Professor Titular da Escola de Enfermagem da Universidade de São Paulo
} 
intelectuais, estratégias cognitivas, atitudes e habilidades motoras. No entanto, o que se observa na prática é que a habilidade motora é privilegiada no manuseio de esfigmomanômetros, embora o desempenho profissional clame pelo desenvolvimento das demais capacidades.

Foi considerando os problemas concernentes à medida da PA, especialmente a ausência de programas educativos específicos, a insuficiência de literatura nacional que discuta os aspectos críticos da medida, e a relevância do papel do enfermeiro na avaliação da PA, que nos propusemos a realização de estudo com o objetivo de identificar o embasamento teórico que fundamenta a prática da medida da pressão arterial e caracterizar o conhecimento do enfermeiro quanto aos aspectos conceituais e fatores anátomo-fisiológicos que influenciam na medida da pressão arterial, visando contribuir para a construção de programas de educação continuada e para a atualização deste conhecimento.

\section{MATERIAL E MÉTODO}

O estudo foi desenvolvido em três hospitais da cidade de São Paulo, especializados em cardiologia, com Unidades de Terapia Intensiva e mantenedores de cursos de aprimoramento ou residência de enfermagem. Estes critérios visaram selecionar profissionais que tivessem conhecimento sobre a mensuração da PA, já que os enfermeiros que trabalham com clientes portadores de alterações cardiovasculares executam o procedimento com grande freqüência, precisando fornecer dados precisos que sirvam de parâmetros para o estabelecimento e avaliação de condutas médicas e de enfermagem.

A coleta de dados, foi realizada no mês de março de 1994, em dias marcados aleatoriamente com as chefias dos respectivos serviços.

O instrumento de coleta de dados constou de formulário (Anexo 1) com 10 itens relativos aos aspectos conceituais e os fatores anátomo-fisiológicos que influenciam a medida da PA.

Foram elaboradas questões abertas e fechadas que possibilitassem respostas diretas e objetivas, as quais foram efetuadas com base em levantamento e análise cuidadosa da literatura concernente ao assunto, além de parte relativa à caracterização da amostra em relação ao tempo de graduação, tempo de exercício profissional, cursos de pós-graduação realizados, setor ou unidade de atuação e fontes possíveis do conhecimento sobre PA. O levantamento da literatura baseou-se nas recomendações da American Heart Association (AMERICAN HEART ASSOCIATION AND THE CARDIAC SOCIETY OF GREAT BRITAIN AND IRELAND, 1939; BORDLEY III et al., 1951; KIRKENDALL et al., 1967;
KIRKENDALL et al., 1980; FROHLICH et al., 1988; PERLOFF et al.,1993); além das publicações de destacados periódicos como: Hypertension, Journal of Hypertension, Journal of Advanced Nursing, entre outros, que subsidiaram a elaboração das questões e contribuíram também para a análise dos dados obtidos.

\section{ANÁLISE DOS RESULTADOS}

Foram entrevistados 84 enfermeiros, sendo 30 da instituição A; 14 da $\mathrm{B}$ e 40 da $\mathrm{C}$. Estes números correspondem a cerca de $70 \%$ dos enfermeiros presentes durante o dia escolhido para a coleta dos dados. A maioria $(56,0 \%)$ dos entrevistados tinha igualmente de 0 a 5 anos de formação e de trabalho na enfermagem; $82,1 \%$ tinham cursos de especialização; $7,1 \%$ tinham mestrado e apenas 1,2\% fazia doutorado. Quanto à área de atuação profissional, a mais citada foi Unidade de Internação $(55,1 \%)$, seguida por Unidade de Terapia Intensiva $(35,2 \%)$. As fontes de aquisição do conhecimento relativo à medida da pressão arterial foram: cursos de graduação (96,4\%), exercício profissional (72,6\%), leituras (53,6\%) e os cursos de especialização $(50,0 \%)$. Três enfermeiros, curiosamente, não citaram o seu curso de graduação como fonte de conhecimento.

A seguir serão discutidas as respostas das questões relativas ao objetivo proposto, procurando analisá-las de acordo com os dados apresentados na literatura específica.

O conhecimento dos enfermeiros sobre os aspectos conceituais da pressão arterial e fatores anátomo-fisiológicos que influenciam a medida, foi investigado por meio de três questões. Nas Tabelas 1, 2 e 3 estão apresentados os resultados encontrados.

Tabela 1 - Conceitos de pressão arterial, segundo os enfermeiros. São Paulo, 1994

\begin{tabular}{lcc}
\hline \multicolumn{1}{c}{ CONCEITOS } & TOTAL & $\%$ \\
\hline $\begin{array}{l}\text { Pressão do sangue na parede } \\
\text { arterial }\end{array}$ & 40 & 47,6 \\
$\begin{array}{l}\text { Pressão de bombeamento do } \\
\text { coração }\end{array}$ & 09 & 10,7 \\
PAS e PAD & 09 & 10,7 \\
Outras & 17 & 20,3 \\
Não responderam & 09 & 10,7 \\
\hline TOTAL & 84 & 100,0 \\
\hline
\end{tabular}


Tabela 2 - Parâmetros que são avaliados quando se mede a PA, segundo os enfermeiros. São Paulo, 1994

\begin{tabular}{lcc}
\hline \multicolumn{1}{c}{ PARÂMETROS } & \multicolumn{2}{c}{ TOTAL } \\
\hline PAS e PAD & 21 & $\%$ \\
Resistência vascular periférica & 16 & 14,9 \\
Débito cardiaco & 14 & 12,6 \\
Força de ejeção e relaxamento cardiaco & 11 & 9,9 \\
Fatores hemodinâmicos & 05 & 4,5 \\
Outros & 33 & 29,7 \\
Não responderam & 11 & 10,0 \\
& & \\
\hline TOTAL* & \multirow{2}{*}{111} & 100,0 \\
\hline * Allguns
\end{tabular}

* Alguns enfermeiros citaram mais de um parâmetro

Tabela 3 - Aspectos anátomo-fisiológicos que influenciam a medida da PA, segundo os enfermeiros entrevistados. São Paulo, 1994

\begin{tabular}{llc}
\hline \multicolumn{1}{c}{ ASPECTOS } & \multicolumn{2}{c}{ TOTAL } \\
& N & $\%$ \\
\hline Estado emocional & 22 & 17,6 \\
Posição do cliente & 22 & 17,6 \\
Condições nutricionais (peso e dieta) & 20 & 16,0 \\
Atividades fisicas & 10 & 8,0 \\
Locais diferentes de medida da PA & 08 & 6,4 \\
Outros & 43 & 34,4 \\
& & \\
\hline \multirow{2}{*}{ TOTAL* } & 111 & 100,0 \\
\hline
\end{tabular}

* Alguns enfermeiros relacionaram mais de um aspecto

O conceito de pressão arterial mais citado pelos entrevistados foi: pressão do sangue nas artérias, especificamente na parede arterial (47,6\%). Mesmo que outros conceitos tenham sido expressos, como a pressão arterial representa a força com que o coração bombeia o sangue para os vasos $(10,7 \%)$ e é a pressão arterial sistólica e a pressão arterial diastólica $(\mathbf{1 0 , 7 \%})$, verifica-se que o conceito correto de pressão arterial é conhecido por um número significante de enfermeiros.

Os entrevistados relacionaram vários parâmetros que estariam sendo avaliados quando da verificação da PA, sendo os mais citados a pressão arterial sistólica e a pressão arterial diastólica $(\mathbf{1 8 , 9 \% )}$, a resistência vascular periférica $(14,4 \%)$ e o débito cardíaco (12,6\%). Quanto aos aspectos anátomo-fisiológicos que poderiam influenciar a medida da $\mathrm{PA}$, os mais lembrados foram: o estado emocional do cliente $(17,6 \%)$, a posição do cliente durante a medida $(17,6 \%) e$ condições nutricionais relacionadas ao peso e à dieta que costumam ingerir $(16,0 \%)$. A prática de exercícios físicos foi menos citada $(8,0 \%)$.

Para GUYTON (1984) pressão é a força exercida pelo sangue circulante contra qualquer área unitária da parede vascular. Esse mesmo conceito é adotado por ROMERO (1968), que considera ser a pressão sangüínea aquela transmitida pelo sangue circulante às paredes das artérias, relacionando cinco fatores determinantes da PA: débito cardíaco e energia da contração ventricular; quantidade de sangue circulante, volemia; qualidade do sangue e viscosidade sangüínea; elasticidade das artérias ou resistência central; tonicidade e permeabilidade das arteríolas pré-capilares ou resistência periférica.

GUYTON (1984) considera que a PA tem uma relação básica com o débito cardíaco e a resistência periférica total, que pode ser representada como: Pressão arterial $=$ Débito cardíaco + Resistência periférica total. Em 1985, o mesmo autor adiciona mais um fator, a pressão do átrio direito (PAD), de forma que $\mathrm{PA}=\mathrm{DC} \mathrm{x}$ RPT + PAD.

Monitorando as modulações da pressão arterial, observa-se que os níveis não são estáticos e apresentam uma variabilidade que pode ser decorrente de diversos fatores anátomo-fisiológicos, dentre eles o ritmo circadiano e a atividade física e mental do indivíduo (PICKERING, 1990). Os estudos relacionados ao ritmo circadiano da PA têm demonstrado valores mais elevados pela manhã, que vão declinando à tarde e chegam a níveis mais baixos durante o sono. $\mathrm{O}$ mesmo autor relacionou diversas atividades que levam a alterações transitórias da PA observando elevações que chegaram até $20 \mathrm{mmHg}$ em indivíduos que foram controlados enquanto comiam, trabalhavam, faziam uso de transportes, andavam, vestiam roupas, realizavam tarefas domésticas e telefonavam.

Alterações mais permanentes e profundas dos valores da PA são causadas por atividades físicas e estresse prolongados. Isto é, indivíduos submetidos a trabalhos que causem estresse mental têm alterações permanentes da pressão arterial, estando já bem comprovada a relação entre hipertensão arterial e atividade econômica exercida (RIBEIRO et al., 1981). A idade é outro fator que leva a variações nos valores da pressão arterial, sendo mais baixos na criança e mais elevados no idoso (GUYTON, 1984).

Procurou-se também saber o conhecimento dos enfermeiros sobre a influência da posição do cliente (em pé, sentado e deitado dorsal ou lateralmente) nos valores da PA. Apenas um enfermeiro considerou que, 
independentemente da posição do cliente, os valores da PA seriam sempre iguais.

Indagados sobre a forma de ocorrência da variação dos valores, os entrevistados deram as respostas que estão apresentadas nas Tabelas 4 e 5 .

Tabela 4 - Posição do cliente que favorece valores mais elevados da PA, segundo os enfermeiros. São Paulo, 1994

\begin{tabular}{|c|c|c|}
\hline \multirow[b]{2}{*}{ POSIÇÃO } & \multicolumn{2}{|c|}{ TOTAL } \\
\hline & $\mathrm{N}$ & $\%$ \\
\hline Em pé & 68 & 56,2 \\
\hline Sentado & 30 & 24,8 \\
\hline Em decúbito dorsal & 11 & 9,1 \\
\hline Em decúbito lateral & 09 & 7,4 \\
\hline Não sabem/não responderam & 03 & 2,5 \\
\hline
\end{tabular}

TOTAL*

121

100,0

* Alguns enfermeiros relacionaram mais de uma posição

Tabela 5 - Posição que favorece valores mais baixos da PA, segundo os enfermeiros. São Paulo, 1994

\begin{tabular}{|c|c|c|}
\hline \multirow[b]{2}{*}{ POSIÇÄO } & \multicolumn{2}{|c|}{ TOTAL } \\
\hline & $\mathrm{N}$ & $\%$ \\
\hline Em decúbito dorsal & 61 & 53,5 \\
\hline Sentado & 21 & 18,4 \\
\hline Em decúbito lateral & 14 & 12,3 \\
\hline Empé & 12 & 10,6 \\
\hline Não sabem/não responderam & 05 & 4,4 \\
\hline TOTAL* & 113 & 100,0 \\
\hline
\end{tabular}

* Alguns enfermeiros relacionaram mais de uma posição

De acordo com a literatura, mesmo leituras consecutivas no mesmo membro e posição fornecem valores diferentes, mas diferenças de até $5 \mathrm{mmHg}$ não são consideradas significativas (RECOMMENDATIONS FOR ROUTINE BLOOD PRESSURE MEASUREMENT BY INDIRECT CUFF SPHYGMOMANOMETRY, 1992). É importante no entanto, que alguns cuidados sejam observados independentemente da posição corporal assumida pelo cliente. Primeiramente deve-se observar um período de repouso de pelo menos 5 minutos na posição e um relaxamento físico e mental, isto é, uma postura relaxada e também verificar a posição do braço a ser utilizado, que deverá estar ao nível do $4^{\mathrm{O}}$.espaço interscostal, para evitar a influência da pressão hidrostática (MITCHELL et al., 1964).

STEIN ( 1946) comparou medidas da PA no braço direito, mantendo-o em diferentes ângulos de abertura em relação ao tórax e observou que quando o braço era hiperaduzido ao longo do eixo do corpo, a pressão era mais baixa que a pressão intra-arterial diretamente medida, efeito que diminuía com a abdução do braço. Estes achados levaram à recomendação de manutenção de um ângulo de abertura, em relação ao corpo, de 45 a 90 graus.

Como a posição sentada favorece a observação desses dois cuidados e a padronização da posição evita ocorrência de erros, essa é a mais recomendada por todos os Comitês (AMERICAN HEART ASSOCIATION AND THE CARDIAC SOCIETY OF GREAT BRITAIN AND IRELAND, 1939; BORDLEY III et al., 1951; KIRKENDALL et al., 1967; KIRKENDALL et al., 1980; FROHLICH et al., 1988; PERLOFF et al., 1993); deve-se lembrar, no entanto, de anotar a posição do cliente durante a medida.

Quando a PA é verificada em gestantes, e tornase necessária a medida na posição deitada, deve-se utilizar o decúbito lateral esquerdo, evitando a compressão dos grandes vasos abdominais, o que pode levar a desconforto e alterações dos valores (PERRY et al., 1991).

Quanto à posição do tórax durante a medida da PA, SCHNEIDER et al. (1971); VIOL et al. (1979) aventaram a hipótese de que, dependendo do tipo de cadeira, poltrona ou da própria postura do indivíduo, a tensão muscular poderia ocasionar alterações na medida da PA, sendo então recomendado que fique relaxado e apoiado no encosto da cadeira ou poltrona, quando na posição sentado.

Em relação à posição das pernas, o único aspecto detectado na literatura é o seu descruzamento, sobretudo nas publicações da American Heart Association (PERLOFF et al.,1993); nos livros-textos de Enfermagem mais utilizados em nossas escolas, não encontramos referências à posição das pernas.

Pelo conceito de variabilidade da PA e demais considerações da literatura aqui apresentadas, pode-se afirmar que é consistente o conhecimento demonstrado pelos enfermeiros ao responder à primeira parte da questão sobre posições, já que não se deve esperar valores iguais até em medidas consecutivas. No entanto, é preciso considerar que as demais respostas apresentadas para o item não permitem afirmar que existe um conhecimento adequado, já que $56,2 \%$ dos enfermeiros correlacionaram valores mais elevados com a posição em pé e $53,5 \%$ os valores mais baixos com o decúbito dorsal.

Com respeito ao braço preferencial para a medida 
da PA, 52,4\% dos enfermeiros indicaram a não necessidade da escolha de um braço específico, mas $46,6 \%$ consideraram que a medida no braço esquerdo é mais fidedigna e apenas $1,2 \%$ indicou o braço direito como tendo valores mais precisos.

Os enfermeiros que consideraram os dois braços adequados justificaram sua opção afirmando que no indivíduo sadio os valores são semelhantes $(15,5 \%)$; aqueles que preferiram o braço esquerdo explicaram que $e ́$ o que tem mais precisão e permite melhor ausculta $(10,7 \%)$ e porque o braço esquerdo está localizado mais próximo do coração $(7,1 \%)$.

A diversidade de respostas deve estar relacionada a diferenciação que os clínicos fazem na prática, sendo clássica a justificativa de que o lado esquerdo favorece anatomicamente por estar próximo ao coração, o que reveste de caráter empírico, considerando que a geração dos sons decorre do fenômeno auscultatório provocado pela compressão e descompressão de um segmento específico do vaso e não do coração. Do mesmo modo seria questionável a afirmação de que o registro deva ser feito no braço direito por representar melhor a verdadeira pressão.

O conceito de que o braço direito oferece dados mais precisos com respeito à pressão sistêmica, chegou a ser aceito por renomados clínicos, possivelmente em decorrência de discussões orais, porém não foi possível detectar na literatura achados que fundamentassem tal conceito.

Os Comitês especializados (AMERICAN HEART ASSOCIATION AND THE CARDIAC SOCIETY OF GREAT BRITAIN AND IRELAND, 1939; BORDLEY III et al., 1951; KIRKENDALL et al., 1967; KIRKENDALL et al., 1980; FROHLICH et al., 1988; PERLOFF et al., 1993) não destacam nenhum braço que favoreça valores mais precisos, mas recomendam que, durante a primeira medida de pressão arterial, sejam efetuadas verificações em ambos os braços e as medidas não devam ter mais de $10 \mathrm{mmHg}$ de diferença. Caso isso ocorra, deve-se escolher o braço que apresenta o valor mais elevado. Estudando 197 indivíduos normotensos e hipertensos, KRISTENSEN \& KORNERUP (1982) não encontraram dominância em nenhum grupo, nem diferenças significativas entre a PA média nos dois braços, mas $49 \%$ dos indivíduos estudados apresentavam variações da PAS iguais ou maiores que $10 \mathrm{mmHg}$, concluíram então que as alterações observadas deveriam estar relacionadas às alterações estruturais das artérias, e recomendaram que o valor mais elevado da pressão arterial deve determinar se o indivíduo é ou não hipertenso.

Como algumas situações clínicas específicas exigem que a pressão arterial seja medida em diferentes locais, foram elaboradas questões específicas para verificar o conhecimento dos enfermeiros sobre locais alternativos para a medida da PA. Procurou-se também saber como os enfermeiros avaliavam sua segurança para realizar esse procedimento. Nas Tabelas 6 e 7 estão apresentadas as respostas obtidas.

Tabela 6 - Locais alternativos para a medida da PA, segundo os enfermeiros. São Paulo, 1994

\begin{tabular}{lcc}
\hline \multirow{2}{*}{ LOCAIS ALTERHATIVOS } & \multicolumn{2}{c}{ TOTAL } \\
\hline Mermbros inferiores & N & $\%$ \\
Coxa & 52 & 61,9 \\
Artérias pediosas e popliteas & 10 & 11,9 \\
Artérias tibiais & 05 & 5,9 \\
Artérias popliteas e tibiais & 05 & 5,9 \\
Panturrilha & 03 & 3,7 \\
Pé & 02 & 2,4 \\
Outros & 02 & 2,4 \\
& 05 & 5,9 \\
\hline \multirow{2}{*}{ TOTAL } & 84 & 100,0 \\
\hline
\end{tabular}

Tabela 7 - Avaliação da segurança para utilização de locais alternativos para a medida da PA, segundo os enfermeiros. São Paulo, 1994

\begin{tabular}{lcc}
\hline & \multicolumn{2}{c}{ TOTAL } \\
AVALIAÇÃO DA SEGURANÇA & $\mathrm{N}$ & $\%$ \\
\hline Têm segurança e experiência & 39 & 46,4 \\
São totalmente inseguros & 31 & 36,9 \\
Têm dúvidas sobre os valores & 14 & 16,7 \\
corretos & & \\
\hline TOTAL & 84 & 100,0 \\
\hline
\end{tabular}

Analisando os dados apresentados, observa-se que os enfermeiros sabem que os membros inferiores podem ser utilizados para a verificação da $\mathrm{PA}$, sendo que a maioria $(61,9 \%)$ os indicou como locais alternativos, mesmo sem denominar as artérias que utilizariam; $12,0 \%$ sugeriram que se utilizasse a coxa e $6,0 \%$ as artérias pediosas, poplíteas e tibiais. O comportamento predominante, ou seja, de não identificar artérias era esperado, já que se fala em verificação da PA no braço e não na artéria braquial.

Complementando as respostas, 46,4\% dos 
entrevistados responderam que tem experiência e segurança para medir a PA em locais alternativos, pois realizam com freqüência esse procedimento. Em contraposição, $36,9 \%$ nunca tiveram oportunidade de utilizar locais alternativos, e sentem-se inexperientes e inseguros; $16,7 \%$ afirmaram não conhecer os valores corretos para a PA quando verificada em outras artérias, o que impede que se sintam muito seguros.

Alguns enfermeiros justificaram suas respostas comentando que utilizam outros locais quando há impossibilidade de uso dos membros superiores por lesões locais; ausência de pulso braquial; dificuldade de ausculta e mesmo para esclarecimento diagnóstico. A maior parte dos enfermeiros considera que os valores da PA medidos na artéria braquial e em outras artérias são semelhantes.

Apesar de não ter sido citado pelos entrevistados, o antebraço pode ser utilizado como alternativa para braços obesos quando não existem manguitos suficientemente largos para a medida precisa. FORSBERG et al. (1970), no entanto, encontraram diferenças entre as medidas realizadas no braço e antebraço; esses achados foram parcialmente confirmados por TACHOVSKY (1985) que faz algumas recomendações: que a medida no antebraço seja evitada quando a pressão for imprescindível para a tomada de decisão importante, e que quando houver necessidade de ser medida a PA no antebraço, alguns aspectos devem ser considerados como: precisão na identificação do local a ser colocado o estetoscópio; uso de manobras para aumentar a audibilidade dos sons e a ressalva de que a PAS no antebraço pode ser menor que no braço e a PAD maior.

A utilização da perna é indicada nas avaliações dos distúrbios vasculares periféricos ou quando existe impossibilidade de utilização dos braços por terapia intravenosa ou cirurgia local. HOCKEN (1967) estudou 30 pacientes registrando e comparando os valores médios da PA nas artérias braquial, poplítea e tibial posterior. Constatou que os valores encontrados na tibial posterior eram apenas 1,4 mmHg inferiores que os valores médios na artéria braquial. Para verificação dos valores da PA na coxa (artéria poplítea) foi utilizado um manguito mais largo que o padrão $(17 \times 57 \mathrm{~cm})$, e os pacientes foram colocados em pronação. A discrepância entre os valores médios encontrados na poplítea e na braquial foram de $12 \mathrm{a}+26 \mathrm{mmHg}$, com uma média de $+8,4 \mathrm{mmHg}$. O autor considerou ser a utilização da perna mais satisfatória do que a da coxa, em razão da melhor correlação com os valores da braquial, da possibilidade de ser usado o manguito padrão, da maior facilidade para a detecção dos sons de Korotkoff e pela posição supina, mais confortável para o paciente.

Resumindo os aspectos abordados na literatura, a utilização de locais alternativos deve ser feita quando a medida da PA é imprescindível e as braquiais estão impossibilitadas por presença de catéteres ou lesões locais, mas atendendo aos aspectos levantados e desde que no registro dos valores fique bem claro o local onde foram obtidos.

Os enfermeiros foram questionados sobre o significado da expressão pressão arterial de repouso, por ser um termo conceitual comum nos compêndios de fisiologia, nos textos referentes às alterações dos níveis de PA e nos levantamentos de dados de clientes em geral ou especificamente dos portadores de hipertensão arterial. Esta questão foi complementada solicitando-se que informassem qual o número de medidas necessárias para se determiná-la. As respostas obtidas estão expressas nas Tabelas 8 e 9.

Tabela 8 - Significado da expressão pressão arterial de repouso, segundo os enfermeiros. São Paulo, 1994

\begin{tabular}{lcc}
\hline \multicolumn{1}{c}{ SIGNIFICADO } & \multicolumn{2}{c}{ TOTAL } \\
\hline PA verificada após repouso físico & 31 & 36,9 \\
Verificada após repouso físico e & 24 & 28,6 \\
mental & & \\
Verificada com o cliente deitado & 11 & 13,1 \\
Valores normais da PA & 02 & 2,4 \\
Não sabeminão responderam & 16 & 19,0 \\
& & \\
TOTAL & 84 & 100,0 \\
\hline
\end{tabular}

Tabela 9 - Número de medidas para determinação da pressão arterial de repouso, segundo os enfermeiros. São Paulo, 1994

\begin{tabular}{lcc}
\hline \multicolumn{1}{c}{ NÚMERO DE MEDIDAS } & N & $\%$ \\
\hline Verificar PA três vezes & 16 & 19,0 \\
Verificar PA duas vezes & 12 & 14,3 \\
Verificar PA uma vez & 09 & 10,7 \\
VerificarPA três vezes em momentos & 07 & 8,3 \\
diferentes & & \\
Outros & 07 & 8,3 \\
Não sabem/não responderam & 33 & 39,4 \\
\hline \multirow{2}{*}{ TOTAL } & 84 & 100,0 \\
\hline
\end{tabular}


Para $31(36,9 \%)$ enfermeiros a pressão arterial de repouso é aquela verificada após o cliente permanecer em repouso físico por um determinado período de tempo; $24(28,6 \%)$ enfermeiros indicaram que o repouso deveria ser físico e mental; $11(13,1 \%)$ consideraram ser a PA medida com o cliente deitado, e $16(19,0 \%)$ enfermeiros não conheciam esta expressão.

Com respeito ao número de vezes da medida da PA necessário para a determinação da pressão arterial de um cliente, $16(19,0 \%)$ enfermeiros responderam, que deveria verificar a PA 3 vezes; 7 (8,3\%) enfermeiros especificaram que as três medidas deveriam ser realizadas em momentos diferentes e $33(39,4 \%)$ não souberam como se determinava a pressão arterial de repouso.

O conhecimento da maior parte dos enfermeiros sobre a expressão é correto, e é possível que os que responderam ser $a$ pressão do cliente deitado, tenham confundido o termo repouso, correlacionando-o com repouso no leito, situação rotineira nos hospitais de cardiologia. De qualquer forma, quase metade dos entrevistados não soube o significado, sendo que 16 $(19,0 \%)$ deles não responderam à questão.

Os fisiologistas consideram que a PA de repouso eqüivale à pressão basal, e deve ser obtida após um período de suspensão de estímulos físicos, metabólicos, mentais e emocionais (KIRKENDALL et al., 1967). Um repouso de 5 minutos na posição de medida é recomendado, além de orientar para não fumar, comer, e até mesmo falar.

Quanto ao número de vezes que a PA deve ser verificada a Organização Mundial de Saúde recomenda que, para o diagnóstico de hipertensão sejam repetidas no mínimo 2 vezes com intervalos de não menos de 3 minutos (HYPERTENSION NEWS, 1993).

Nos protocolos de medida indireta da PA a indefinição quanto ao número de medidas tem sido um dos problemas metodológicos enfrentados. Enquanto alguns autores como WILBER \& BORROW (1972) preferem a média das três leituras, outros, como ALMEIDA et al. (1979) optam pelo registro do menor dos três valores, considerando a vantagem de ambientação na diminuição do nível de estresse causado pelo procedimento. FAGAN et al. (1988) compararam medidas duplas e triplas, realizadas com pequeno intervalo de tempo com medida única e concluíram que, quando a medida é realizada observando os cuidados de posição e relaxamento do cliente, os resultados obtidos são fidedignos; a ocorrência de viés é mais comum nas medidas tríplices por fadiga do procedimento. No estudo de ARCURI (1985) os resultados obtidos permitiram concluir que após 5 minutos de repouso orientado com relaxamento muscular, excetuando a medida inicial, a partir da segunda medida ocorreram apenas pequenas diferenças, isto é, entre a primeira e a segunda medida a média foi de $1,7 \mathrm{mmHg}$; já entre a segunda e a terceira foi de $0,1 \mathrm{mmHg}$, e a mesma média foi encontrada entre a terceira e a quarta verificação.

\section{ALGUMAS CONSIDERAÇÕES}

Pelos resultados apresentados observou-se que os enfermeiros têm desconhecimento e dúvidas sobre diversos aspectos conceituais e fatores anátomofisiológicos que influenciam a medida da pressão arterial, assim faz-se necessário providências, quer a nível da graduação ou mesmo em cursos de educação continuada, que possam contribuir para diminuir as lacunas do conhecimento.

Para a realização deste estudo várias questões metodológicas foram consideradas, pois analisar e caracterizar um conhecimento não é fácil, uma vez que requer uma seleção cuidadosa de aspectos que sejam considerados essenciais para o desempenho de uma atividade, no caso específico, a medida da pressão arterial. Além disto, a discussão de aspectos cognitivos tem caráter polêmico. Qual o conhecimento ideal que poderia ser esperado? A totalidade das respostas corretas? Com base na avaliação acadêmica rígida, como ocorre em algumas universidades no exterior e até nas diferenciadas no Brasil, este valor seria de $70 \%$, ou $50 \%$ que é a realidade no nosso meio? Como seria a avaliação de outros procedimentos na área da enfermagem? E nas demais áreas? A observação empírica tem demonstrado que outros profissionais que também executam este procedimento, a medida da PA, não estão atualizados com relação as fronteiras do conhecimento em esfigmamonometria.

Mesmo que os resultados apresentados não possam ser generalizados, porque representam o conhecimento de um grupo específico, espera-se que os mesmos sirvam para subsidiar o diagnóstico do perfil dos enfermeiros, além de possibilitar o estabelecimento de programas de educação continuada, voltados para o aperfeiçoamento e atualização do conhecimento da medida indireta da pressão arterial.

Ao retratar resultados não tão satisfatórios, a expectativa das autoras diante do levantamento bibliográfico e da discussão dos dados é estar contribuindo com material atualizado para a realização de programas de educação continuada e para o ensino nos cursos de graduação em enfermagem. 
INFLUENCE OF THE ANATOMICAL AND PHYSIOLOGICAL FACTORS IN THE INDIRECT MEASUREMENT OF BLOOD PRESSURE: IDENTIFICATION OF NURSING KNOWLEDGE

The study was developed by clinical nurses specialized in cardiology and had the purpose to characterize the kwowledge about the conceptual aspects and the anatomical and physiological factors that interfere in the marks of blood pressure.

KEY WORDS: indirect measurement of blood pressure, sphygmomanometry

\section{INFLUENCIA DE LOS RASGOS ANÁTOMO-FISIOLÓGICOS EN LA MENSURACIÓN INVERSA DE LA PRESIÓN ARTERIAL: IDENTIFICACIÓN DEL CONOCIMIENTO DE LOS ENFERMEROS}

El estudo fué realizado con enfermeros de hospitales de cardiología y el objetivo fue caracterizar el conocimiento respecto a los rasgos conceptuales y anatomo-fisiológicos que influyen en la mensuración de la presión arterial.

TÉRMINOS CLAVES: mensuración de la presión arterial, esfingonometría

\section{ANEXO 1}

\section{CONHECIMENTO DO ENFERMEIRO SOBRE A MEDIDA DA PRESSÃO ARTERIAL Data: Instituição:}

\section{A: DADOS DE IDENTIFICAÇÃO}

A-1. Ano da graduação em enfermagem:

A-2. Curso de habilitação: não:

sim: __ Em que área?

A-3. Curso de especialização: não: __ sim: —_ ainda em curso:

A-4. Curso de mestrado: não: __ sim: __ _ ainda em curso: ___ Em que área?

A-5. Curso de doutorado: não: _ـ sim: __ ainda em curso:__ Em que área?

A-6. Unidade de trabalho:

A-7. Tempo de trabalho na instituição:

A-8. Fontes do seu conhecimento sobre pressão arterial (podem ser indicadas várias fontes): graduação:__ habilitação:__ especialização: __ mestrado: __ doutorado: exercício profissional:_— outros profissionais (enfermeiros ou não): leitura de livros ou periódicos sobre o assunto:__ cursos específicos: outros:

B: QUESTÕES SOBRE A MEDIDA DA PRESSÃO ARTERIAL

1. Como você conceitua pressão arterial?

2. Que parâmetros estão sendo avaliados quando você verifica uma PA?

3. Que aspectos anatômicos e fisiológicos podem influenciar a medida da PA?

4. O que podemos esperar, em relação aos valores da pressão arterial sistólica (PAS) e da pressão arterial diastólica (PAD), segundo as diferentes posições do cliente durante a medida (em pé, sentado, deitado em posição lateral, deitado em posição dorsal):

- valores iguais em todas as posições: não: sim:

- caso negativo, relacione as posições que levam a valores mais altos da PA e as posições que levam a valores mais baixo da PA

5. Você tem preferência pelo braço direito ou esquerdo para verificar a PA? não: $\operatorname{sim}:$ Justifique sua resposta.

6. Que outros lugares além da artéria braquial, podem ser utilizados para verificar a PA?

7. Você tem segurança para verificar a PA em outro local? Justifique sua resposta.

8. Em que situações recorre-se a locais diferentes da artéria braquial para verificar a PA?

9. O que significa a expressão: pressão arterial de repouso?

10. Qual o número de medidas necessárias para se determinar a PA de repouso de um cliente? 


\section{REFERÊNCIAS BIBLIOGRÁFICAS}

01. AMERICAN HEART ASSOCIATION AND THE CARDIAC SOCIETY OF GREAT BRITAIN AND IRELAND. Standardization of blood pressure readings. Am.Heart J., v.18, p.95-101, 1939.

02. ARCURI, E.A.M. Estudo comparativo da medida indireta da pressão arterial com manguito de largura correta e com manguito de largura padrão. São Paulo, 1985. Tese (Doutorado) Instituto de Ciências Biomédicas, Universidade de São Paulo.

03. BORDLEY III, J.J. et al. Recommendations of human blood pressure determinations by sphygmomanometers. Circulation, v.4, p.503-9, 1951.

04. ALMEIDA, D.B.de. et al. Níveis pressóricos de estudantes de 16 a 25 anos, da área urbana do município de Botucatu, São Paulo, em 1975. Bol. Of. Sanit. Panam., v.87, p.405-13, 1979.

05. FAGAN, T.C. et al. Single versus triplicate measurements of blood pressure and hearty rate. Hypertension, v.11, n.3, p.282-4, 1988.

06. FORSBERG, S.A . et al. Validity of blood pressure measurement with cuff in the arm and forearm. Acta Med. Scand. , v.188, p.389-96, 1970.

07. FROHLICH, E.D. et al. Recommendations for human blood pressure determination by sphymomanometers. Circulation, v.77, p.501A14A, 1988.

08. GAGNÉ, L.M. Princípios essenciais da aprendizagem para o ensino. Porto Alegre: Globo, 1980.

09. GUYTON, A .C. Tratado de fisiologia médica. 6 . ed. Rio de Janeiro: Interamericana, 1984. p. 199223.

10. HOCKEN, A.G. Measurement of blood pressure in the leg. Lancet, v.4, p.1241-3, 1967.

11. HYPERTENSION News. Bulletin of the International Society of Hypertension, Jun.1993. p.1-5.

12. KIRKENDALL, W.M. et al. Recommendations for human blood pressure determination by sphygmomanometers. Circulation, v.36, p.9808, 1967.

13. KIRKENDALL, W.M. et al. Recommendations for human blood pressure determination by sphygmomanometers: subcommitee of the AHA postgraduate education committee. Circulation, v. 62, p.1146A -55A, 1980.
14. KRISTENSEN, B.O.; KORNERUP, H.J. Which arm to measure the blood pressure? Acta. Med. Scand., v. 670, p. 69-73, 1982. Supplement.

15. MANCIA, G. et al. Alerting reaction and rise in blood pressure during measurement by physician and nurse. Hypertension, v.9, p. 200-15, 1987.

16. MITCHELL, P.L. et al. Effect of vertical displacement of the arm on indirect blood pressure measurement. N.Engl.J.Med., v.27, p.72-4, 1964.

17. PERLOFF, D. et al. Human blood pressure determination by sphygmomanometry. Circulation, v. 88, n.5, p.2460-70, 1993. Part.I.

18. PERRY, I.J. et al. Conflicting views on the measurement of the blood pressure in pregnancy. Brit. J. Obst. Gynecol., v. 98, p. 241-3, 1991.

19. PICKERING, T.G. Physiological aspects of noninvasive ambulatory blood pressure monitoring. NIPS, v. 5, p.176-9, 1990.

20. PIERIN, A .M.G. Medidas da pressão arterial no ambulatório pelo cliente, enfermeira e médico comparados a registros domiciliares. São Paulo, 1992. 119p. Tese (Doutorado) - Escola de Enfermagem, Universidade de São Paulo.

21. RECOMMENDATIONS for routine blood pressure measurement by indirect sphygmomanometry. Am. J. Hypertension, v. 5, n. 4, p. 207-9, 1992.

22. RIBEIRO, M.B. et al. Hypertension and economic activities in São Paulo, Brazil. Hypertension, v. 3, p. 233-7, 1981.

23. ROMERO, V. Semiologia médica. 11 ed. Rio de Janeiro: Guanabara, 1968. v. 1, p. 344.

24. SCHNEIDER, R. A. et al. Arterial pressures recorded in hospital and during ordinary daily activities: contrasting data in subjects with and without ischemic heart disease. J. Chronic Dis., v. 23, p. $647-57,1971$

25. STEIN, I. The effect of change of position of the arm upon blood pressure. Am. Heart J., v. 31, p. 47780, 1946.

26. TACHOVSKY, B.J. Indirect auscultatory blood pressure measurement at two sites in the arm. Res. Nurs. Health, v. 8, p. 125-9, 1985.

27. VIOL, G. W. et al. Seating as a variable in clinical blood pressure measurement. Am. Heart J., v. 98, p. 813-4, 1979.

28. WILBER, J. A.; BARROW, J. C. Hypertension: a community problem. Am. J. Med., v. 52, p. 653, 1972.

29. WILCOX, J. Observer fators in the measurement of blood pressure. Nurs.Res., v.10, p.4-17, 1961. 